\title{
Alterações clínicas e hematológicas em gatos domésticos naturalmente infectados pelo Vírus da Leucemia Felina (FeLV)
}

\author{
Hematologic and clinical changes in domestic cats naturally infected with Feline Leukaemia Virus \\ (FeLV)
}

\author{
Nádia Rossi de Almeida ${ }^{\dagger *}$, Lidiane de Castro Soares ${ }^{\dagger}$, Amanda Brito Wardini* \\ Resumo
}

O Vírus da Leucemia Felina (FeLV) é um retrovírus imunossupressor e o maior causador de morte dentre as doenças infecciosas felinas. O FeLV induz ao desenvolvimento de distúrbios degenerativos e/ou mieloproliferativos nos animais infectados, que sucumbem à infecções oportunistas devido à imunossupressão. As alterações nos parâmetros hematológicos em gatos FeLV positivos muitas vezes não condizem com o estado clínico do animal, podendo o mesmo ser um portador assintomático apresentando alterações hematológicas, ou sintomático sem alterações nestes parâmetros. O objetivo do presente estudo foi avaliar as alterações nos parâmetros clínicos e hematológicos de gatos domésticos infectados naturalmente pelo FeLV, nas fases sintomáticas e assintomáticas da doença. Desta forma, foram selecionados 40 gatos domiciliados previamente testados como positivos para o FeLV pela técnica de imunofluorescência indireta. O exame físico foi realizado e os animais foram separados em dois grupos: os gatos sintomáticos e os assintomáticos para a infecção. Amostras de sangue para a realização de hemograma foram coletadas de ambos os grupos e o teste estatístico ANOVA foi realizado para a comparação das alterações hematológicas. O exame clínico indicou 37,5\% dos gatos como portadores assintomáticos e $62,5 \%$ sintomáticos, sendo a perda de peso e alterações de mucosas os achados mais frequentes. O hemograma evidenciou anemia e linfopenia como os parâmetros hematológicos que apresentaram diferenças estatísticas entre os dois grupos estudados, sendo que $56 \%$ dos gatos assintomáticos apresentaram anemia. Em face aos resultados encontrados, concluiu-se que gatos FeLV positivos sintomáticos apresentaram alterações hematológicas condizentes com o quadro de imunossupressão clínico.

Palavras-chave: Retrovírus; Imunossupressão; Sinais clínicos; Hematologia.

\begin{abstract}
The Feline Leukaemia Virus (FeLV) is an immunosuppressant retrovirus and leading cause of death among infectious diseases affecting cats. FeLV induces the development of degenerative and/or proliferative disorders in infected animals, who succumb to the disease due to opportunistic infections resulting from immunosuppression. Changes in hematological parameters in FeLV positive cats often do not match the clinical presentation of the animal. In this case the animal could be an asymptomatic carrier presenting hematological changes, or with symptomatic presentation without hematological alterations. The aim of this study was to evaluate changes in clinical and hematological parameters of domestic cats naturally infected with FeLV, in symptomatic and asymptomatic stages of the disease. The study evaluated 40 domestic cats with identified domiciles which had previously tested positive for FeLV by indirect immunofluorescence. Physical examination was performed and the animals were separated into two groups: asymptomatic and symptomatic infection. Blood samples for hematology were collected from both groups and ANOVA was performed to compare the hematological results. Clinical evaluation indicated $37.5 \%$ of the cats as asymptomatic and $62.5 \%$ as symptomatic with weight loss and changes in the mucous membranes the most prevalent findings. The statistical analysis of the hematology results showed that red blood cell count and lymphocyte count were statistically different between the two groups, with $56 \%$ of symptomatic animals showing anemia. In view of the results, it was concluded that symptomatic FeLV positive cats showed hematological results consistent with the observed clinical immunosuppression.

Keywords: Retroviruses; Immunosuppression; Clinical signs; Hematology.
\end{abstract}

\section{Introdução}

A Leucemia Viral Felina é uma doença infecciosa que acomete tanto os felinos domésticos quanto os selvagens e é causada por um retrovírus imunossupressor e oncogênico, que está associado aos distúrbios degenerativos e mieloproliferativos. ${ }^{1,2} \mathrm{O}$ vírus da Leucemia Felina (FeLV) está amplamente disseminado ao redor do mundo e a ocorrência da infecção está associada aos fatores de risco e às medidas de controle, representando a maior causa de morte dentre as doenças infecciosas dos felinos. ${ }^{2}$

A transmissão do FeLV ocorre pela via oronasal através do contato íntimo e prolongado entre um gato infectado e um gato sadio, principalmente por intermédio da saliva rica em partículas virais. ${ }^{3,4} \mathrm{O}$ FeLV pode ser transmitido por via transplacentária além de estar presente em outros fluidos corporais como na lágrima,

Afiliação dos autores:† Universidade Severino Sombra, Pró-Reitoria de Ciências da Saúde e Humanas, Curso de Medicina Veterinária, Vassouras-RJ, Brasil.

\$ Curso de Pós-Graduação em Bioquímica e Imunologia da Universidade Federal de Minas Gerais (UFMG), Belo Horizonte-MG, Brasil.

* Endereço para correspondência: Universidade Severino Sombra, Av. Exped. Oswaldo de Almeida Ramos, 280 - Centro - Vassouras, RJ - CEP $27700-000$.

E-mail: nadiarossialmeida@gmail.com 
plasma, urina e leite. Há a possibilidade de transmissão iatrogênica, como o uso de instrumentos contaminados e transfusão sanguínea. ${ }^{5,6}$

A infecção persistente pelo FeLV é caracterizada por imunossupressão e os gatos frequentemente sucumbem à doenças associadas dentro de meses ou poucos anos. ${ }^{7,4} \mathrm{O}$ FeLV se replica rapidamente nas células em mitose do sistema imune, incluindo linfócitos, neutrófilos, monócitos e macrófagos. Doenças hematopoiéticas, particularmente citopenias causadas pela supressão da medula óssea são achados comuns em gatos FeLV positivos, e incluem anemia (principal complicação não-neoplásica), leucopenia, trombocitopenia, leucemia e doenças reumáticas. ${ }^{6,8}$ A leucopenia é representada principalmente pela neutropenia e linfopenia. O principal mecanismo que leva a neutropenia é o efeito citopático sobre as células precursoras dos neutrófilos (CFU-GM). ${ }^{9}$ As proteínas do FeLV são expressas pelas CFU-GM, onde a resposta de anticorpos anti-FeLV lisam essas células. Gatos com infecções persistentes e latentes podem apresentar um número suficiente de neutrófilos na circulação, porém podem estar com sua função prejudicada. ${ }^{10}$ Além disso, os neutrófilos de gatos infectados têm decréscimo progressivo da atividade fagocitária se comparados com os de gatos não infectados. Já a linfopenia é representada pelo decréscimo de linfócitos $\mathrm{T} \mathrm{CD}^{+}$, o que resulta em uma razão invertida de $\mathrm{CD} 4 / \mathrm{CD} 8$, embora haja significativo decréscimo dos linfócitos $\mathrm{T} \mathrm{CD} 8^{+}$na fase final da doença. ${ }^{7,4,11}$

Os sinais clínicos associados à infecção são variáveis, no entanto, a maioria dos gatos desenvolve perda de peso, desidratação, anorexia, prostração, complexo estomatite-gengivite, neuropatias, distúrbios reprodutivos e diversas infecções concomitantes, incluindo a infecção pelo vírus da imunodeficiência felina (FIV), complexo respiratório viral felino, Toxoplasmose e micoplasmose hemotrópica (Mycoplasma haemofelis). ${ }^{12,13,14}$ Distúrbios mieloproliferativos, os quais são representados pelos linfomas e leucemias, também estão associados à infecção pelo FeLV. ${ }^{15,16,17}$

O diagnóstico da infecção pelo FELV é realizado pela união do exame clínico, exames laboratoriais complementares, e principalmente, pela realização dos testes sorológicos para detecção do antígeno viral p27, como a prova ELISA, testes rápidos imunocromatográficos e a imunofluorescência indireta (IFI). ${ }^{18,2}$ Além disso, o prova molecular de PCR detecta o DNA proviral do FeLV integrado em células sanguíneas, sendo de grande importância para detectar as infecções latentes em medula óssea, as quais são indetectáveis pelos métodos sorológicos. ${ }^{19,20,21}$

No Brasil, o diagnóstico do FeLV apresenta algumas restrições, como o elevado custo do kit comercial imunocromatográfico para a detecção do antígeno p27. O teste apresenta a necessidade da repetição, em caso do mesmo apresentar um resultado positivo, uma vez que é capaz de detectar uma viremia primária não associada às células, podendo ainda haver a eliminação viral pelo hospedeiro. $\mathrm{O}$ mesmo não ocorre com a prova de IFI a qual detecta somente uma viremia persistente após a infecção da medula óssea, porém sua aplicação ainda é restrita às instituições de pesquisa. ${ }^{16,2}$

O objetivo do presente estudo foi avaliar as alterações clínicas e nos parâmetros hematológicos de gatos domésticos infectados pelo FeLV, nas fases sintomáticas e assintomáticas desta doença.

\section{Material e Métodos}

\section{Animais e colheita de amostras}

Para o presente estudo, foram selecionados 40 gatos domésticos domiciliados, naturalmente infectados pelo FeLV, sem predileção por sexo, raça ou idade. Estes animais foram atendidos em clínicas veterinárias, hospitais veterinários, abrigos ou em seus próprios domicílios.

Amostras de sangue no volume de $3 \mathrm{~mL}$ foram colhidas para a realização do hemograma por punção da veia cefálica e armazenadas em tubos heparinizados sob temperatura de refrigeração e enviadas ao Laboratório de Imunologia e Virologia Veterinária (LIVV) da Universidade Federal Rural do Rio de Janeiro, no prazo de até 24 horas.

Esfregaços de sangue periférico foram obtidos por punção de ponta de orelha. As lâminas foram secas à temperatura ambiente, identificadas e acondicionadas de forma a se evitar a lise celular e também enviadas ao (LIVV) dentro de um prazo de até 7 dias após a confecção dos esfregaços. Logo após o recebimento, as lâminas foram fixadas utilizando-se uma solução de acetona e metanol, na relação de 3:1, em temperatura ambiente durante 20 minutos, com o objetivo de preservar o antígeno viral presente em leucócitos e plaquetas. Após a secagem, as mesmas foram processadas imediatamente ou conservadas a uma temperatura de $-20^{\circ} \mathrm{C}$, por um período de até 7 dias para a realização de testes diagnósticos.

\section{Imunodiagnóstico do FeLV}

A IFI para a detecção do antígeno viral nos esfregaços de sangue periférico foi realizada utilizandose kit comercial (Primary Anti-Feline Leukemia Virus Antiserum for IFA and Anti-goat IgG FITC Conjugate; VRMD, Inc - Pullman, WA/USA) de acordo com as normas do fabricante. Um microscópio de fluorescência OLYMPUS BX41 com epi-iluminação, com a objetiva de 40x, foi utilizado para a leitura da reação. Uma lâmina de esfregaço sanguíneo de um animal sabidamente 
positivo pela IFI foi utilizada como controle positivo da reação. A amostra foi considerada positiva quando leucócitos e plaquetas apresentaram uma fluorescência nítida (Figura 1).

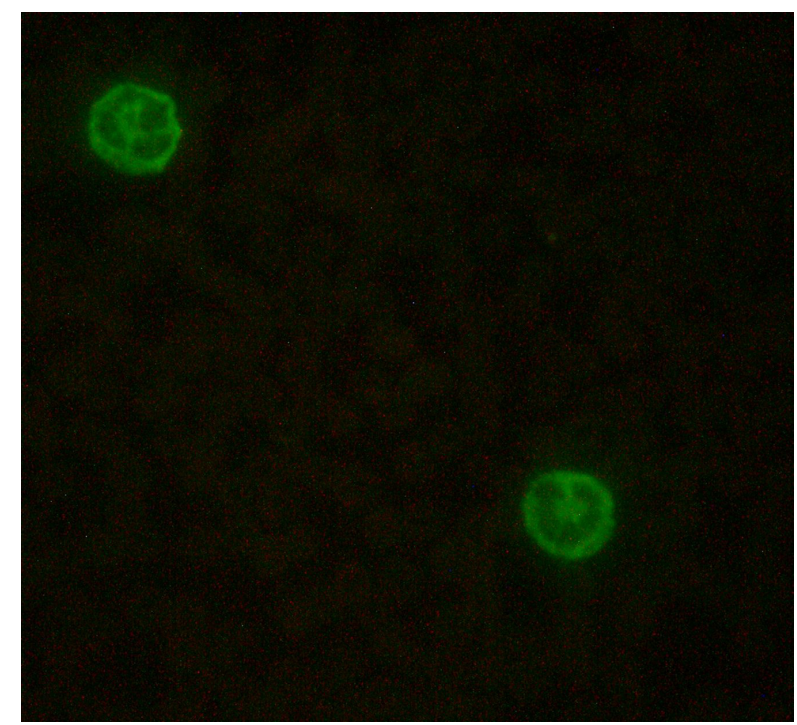

Figura 1. Esfregaço de sangue de um gato FeLV positivo pela IFI apresentando fluorescência em neutrófilos. Aumento de $40 \mathrm{x}$.

\section{Avaliação clínica dos animais}

Para cada animal foi preenchida uma ficha com o histórico e com as possíveis alterações clínicas sugestivas de infecção pelo FeLV, que incluiram: perda de peso, desidratação, alteração das mucosas, linfoadenomegalia, sinais clínicos respiratórios, presença de complexo estomatite-gengivite e presença de massas tumorais sugestivas de linfomas associados ao FeLV. Desta forma, os animais foram divididos em dois grupos, sendo um grupo de gatos sintomáticos e o outro grupo de gatos assintomáticos para a Leucemia Viral felina.

\section{Hemograma}

A determinação do volume globular foi realizada através da técnica micro hematócrito segundo metodologia já descrita na literatura. ${ }^{22} \mathrm{~A}$ contagem de hemácias, plaquetas e leucócitos foram realizadas em hemocitômetro. Hemácias e plaquetas foram diluídas na proporção 1:200 em solução salina $0,9 \%$ e solução de Rees-Ecker, respectivamente. Os leucócitos foram diluídos na proporção 1:20 em líquido de Turk. Os esfregaços sanguíneos foram corados pelo método Panótico Rápido e utilizados para a contagem diferencial de glóbulos brancos por microscopia óptica. A concentração de proteína plasmática total foi determinada por refratometria. A contagem de reticulócitos foi realizada em todos os animais que apresentavam anemia, e para tal utilizou-se $0,1 \mathrm{~mL}$ de sangue o qual foi incubado a $37^{\circ} \mathrm{C}$ juntamente com
0,1mL do corante Azul de Cresil Brilhante durante vinte minutos. Posteriormente foram confeccionados esfregaços, e através de microscopia óptica determinouse o percentual de reticulócitos em relação ao número de 1.000 eritrócitos.

\section{Análise estatística}

Os parâmetros hematológicos foram analisados por Análise de Variância (ANOVA), utilizando-se o programa GraphPad Prisma versão 6 (GraphPad, San Diego, CA, USA) onde as diferenças entre as médias foram consideradas significativas quando $p<0,05$.

\section{Resultados}

\section{Avaliação clínica dos animais}

Todos os animais infectados pelo FeLV foram avaliados clinicamente, sendo que 37,5\% (15/40) dos gatos não apresentaram qualquer sinal clínico sugestivo de Leucemia Viral Felina, sendo aparentemente saudáveis. Dos 62,5\% (25/40) gatos sintomáticos, a perda de peso foi a alteração observada com maior frequência $(48 \%$; 12/25), seguida de alterações de mucosas (hipocoradas ouictéricas), doenças respiratórias e presença de linfomas (3 linfomas mediastínicos, 2 formas extranodais e 1 linfoma multicêntrico). Todas as alterações identificadas no exame clínico podem ser visualizadas na Tabela 1 .

Tabela 1. Sinais clínicos observados nos gatos FeLV positivos avaliados $(\mathrm{n}=40)$

\begin{tabular}{cc}
\hline Sinais clínicos & Frequência \\
\hline Perda de peso & $30,0 \%(12)$ \\
Desidratação & $12,5 \%(5)$ \\
Mucosas hipocoradas ou & $20,0 \%(8)$ \\
ictéricas & $12,5 \%(5)$ \\
Linfoadenomegalia & $17,5 \%(7)$ \\
Doença respiratória & $7,5 \%(3)$ \\
Complexo estomatite- \\
gengivite
\end{tabular}

\section{Alterações Hematológicas}

Uma vez separados os grupos de gatos FeLV positivos em sintomáticos e assintomáticos para a infecção, foi realizada a análise e comparação dos parâmetros hematológicos dos dois grupos (Tabela 2).

A anemia foi observada em 56\% (14/25) dos animais sintomáticos para o FeLV, sendo que em 


\begin{tabular}{|c|c|c|}
\hline \multicolumn{3}{|c|}{$\begin{array}{l}\text { Tabela 2. Parâmetros hematológicos das amostras de sangue dos gatos FeLV positivos avaliados } \\
(\mathrm{n}=40) \text {. }\end{array}$} \\
\hline Parâmetros Hematológicos & Assintomático & Sintomático \\
\hline Hematócrito (\%) & $33,1 \pm 4,06$ b & $22,65 \pm 10,20$ \\
\hline Proteína plasmática total (g/dl) & $7,06 \pm 0,64$ a & $6,91 \pm 0,82$ a \\
\hline Plaquetas (x 103/uL) & $277,9 \pm 120,1^{\text {a }}$ & $222,1 \pm 92,41^{a}$ \\
\hline Leucócitos totais (x 103/uL) & $13,51 \pm 4,22$ & $10,50 \pm 4,08^{\text {a }}$ \\
\hline Neutrófilos (x 103/uL) & $7,22 \pm 2,51$ & $7,39 \pm 6,01^{a}$ \\
\hline Linfócitos (x 103/uL) & $4,25 \pm 2,33 \mathrm{~b}$ & $1,47 \pm 3,20$ b \\
\hline Monócitos (x 103/uL) & $0,59 \pm 0,16^{a}$ & $0,57 \pm 0,20$ \\
\hline Eosinófilos (x 103/uL) & $0,79 \pm 0,66^{a}$ & $0,51 \pm 0,33^{\mathrm{a}}$ \\
\hline $\begin{array}{l}\text { a Dados não apresentam diferença es } \\
{ }^{\mathrm{b}} \text { Dados apresentam diferença estatís } \\
\text { Dados são expressos como média } \pm\end{array}$ & $\begin{array}{l}\mathrm{p}>0,05 \text { entre os } \\
<0,05 \text { entre os grup } \\
8 \text { animais/grupo }\end{array}$ & \\
\hline
\end{tabular}

12 casos apresentou-se normocítica normocrômica. Nos dois casos restantes, a anemia foi responsiva, observando-se a presença de reticulócitos. No grupo assintomático, foi observada anemia em 13.3\% (2/15) dos animais, sendo que os valores se encontraram próximos do valor mínimo de referência de $25 \%$ de hematócrito.

A concentração de proteína plasmática total apresentou-se discretamente acima dos valores de referência em somente um animal do grupo FeLV positivo sintomático. A média da contagem de plaquetas manteve-se dentro da normalidade nos dois grupos estudados, porém, no grupo FeLV positivo sintomático estes valores se aproximaram ao valor mínimo de referência, que é de $200 \times 10^{3} / \mathrm{uL}$.

Apesar da média de leucócitos totais em ambos os grupos se enquadrarem dentro dos valores de referência, foi observada leucopenia em 26,7\% (4/15) dos animais assintomáticos e $32 \%(8 / 25)$ dos animais sintomáticos. A linfopenia foi presente em $20 \%(3 / 15)$ dos animais assintomáticos e em 40\% (10/25) dos animais sintomáticos. A presença de leucocitose foi observada em $12 \%(3 / 25)$ dos gatos FeLV positivos sintomáticos, onde dois animais apresentaram sinais clínicos de infecção respiratória bacteriana secundária ao complexo respiratório viral felino e um apresentou leucemia linfocítica com valores de linfócitos de 86,49 x $10^{3} / \mathrm{uL}$.

Eosinofilia foi observada em $8 \%(2 / 25)$ dos gatos FeLV positivos sintomáticos, os quais apresentaram complexo estomatite-gengivite e a monocitose em um gato com icterícia devido coinfecção pelo hemoparasita Mycoplasma haemofelis.

\section{Discussão}

A crescente aquisição de gatos como animais de companhia e sua concentração em pequenos grupos ou em colônias tem propiciado a incidência e persistência das infecções virais. Assim, o FeLV tornou-se a maior causa de mortes entre os gatos domésticos.

$\mathrm{Na}$ população de gatos avaliados no presente estudo, foi observada uma maior frequência de animais sintomáticos para a infecção. Entretanto, a quantidade de gatos portadores assintomáticos foi expressiva, apontando a importância destes animais como potenciais fontes de infecção. Os sinais clínicos observados nos gatos FeLV positivos sintomáticos estão de acordo com os mais frequentemente citados na literatura, ${ }^{3,16}$ como a perda de peso, desidratação, mucosas hipocoradas ou ictéricas, complexo estomatite-gengivite, doenças respiratórias, bem como a presença de linfomas, que por sua vez estão cada vez mais ocorrentes em gatos FeLV positivos no Brasil. ${ }^{2}$

Pelo fato do FeLV se replicar nas células progenitoras dos eritrócitos na medula óssea, o principal achado clínico-laboratorial em gatos FeLV positivos é a anemia, que geralmente se desenvolve por hemólise, displasia medular, e principalmente, pela hipoplasia ou aplasia eritróide. ${ }^{4,23}$ No presente estudo, a anemia foi observada em 40\% (16/40) dos gatos FeLV positivos, havendo diferença estatística significativa entre as médias de hematócritos do grupo assintomático e sintomático $(\mathrm{p}<0,05)$.

A leucopenia devido à linfopenia é um achado comum em gatos infectados pelo FeLV, principalmente pelo decréscimo de linfócitos $\mathrm{T} \mathrm{CD}^{+}$, o que resulta numa razão $\mathrm{CD} 4 / \mathrm{CD} 8$ invertida. ${ }^{7,4}$ Este achado foi observado em ambos os grupos, com porcentagens de $40 \%$ e $20 \%$ para gatos sintomáticos e assintomáticos, respectivamente, revelando diferença significativa entre os dois grupos estudados. Esta linfopenia causa imunossupressão, o que favorece as infecções oportunistas, como pelos vírus da Rinotraqueíte e Calicivirose felinas, Sporothrix shenckii, Toxoplasma gondii e infecções bacterianas em geral. ${ }^{16,24}$.

A leucocitose neutrofílica está presente em 
aproximadamente $1 / 3$ dos animais virêmicos, em resposta a infecções bacterianas e inflamação. ${ }^{25} \mathrm{Em}$ um estudo previamente realizado ${ }^{26}$ não foram encontradas alterações nas células brancas de animais naturalmente infectados assintomáticos, com exceção de discreta linfopenia. Em outro estudo semelhante ${ }^{27}$ observaram apenas anemia e linfopenia em gatos experimentalmente infectados com FeLV-FAIDS. No presente estudo, a foi observada neutrofilia com desvio à esquerda, principalmente em gatos do grupo FeLV sintomáticos, apesar de não ter apresentado diferença estatística significante.

As alterações hematológicas observadas são condizentes com a infecção persistente pelo FeLV. Contudo, para a realização dos ensaios foram utilizados alguns animais que faziam uso de antiretroviral, antibioticoterapia e/ou quimioterapia. Além disso, estas alterações na avaliação dos parâmetros hematológicos podem não ser exclusivas da infecção pelo FeLV, pois infecções oportunistas e secundárias podem também contribuir para estas alterações, como ocorre em coinfecções pelo Mycoplasma haemofelis, infecções do trato respiratório superior e desenvolvimento de complexo estomatite-gengivite. ${ }^{4,28,24}$

\section{Conclusões}

Os gatos FeLV positivos naturalmente infectados e sintomáticos apresentaram alterações hematológicas condizentes com as relatadas na literatura, onde a anemia e a linfopenia foram as alterações que apresentaram diferenças significativas entre os dois grupos estudados.

Os sinais clínicos observados nos animais estão em consonância com o caráter imunossupressor do vírus, onde a perda de peso e presença de mucosas hipocoradas ou ictéricas foram os achados mais frequentes.

Um número relevante de gatos FeLV positivos assintomáticos foram observados, evidenciando este grupo como um potencial fator de risco para a infecção.

\section{Declarações}

Os autores não possuem conflitos de interesse diretos ou indiretos. A fonte de financiamento desta pesquisa não foi declarada.

\section{Referências}

1. Mochizukia H, Takahashia M, Nishigakib K, Idec T, Goto-koshinoa Y, Watanabe S, Sato H, Kotera Y, Fujino Y, Ohno K, Uchida K, Tsujimoto $\mathrm{H}$. Establishment of a novel feline leukemia virus (FeLV) negative B-cell cell line from a cat with B-cell lymphoma. Veterinary Immunology and Immunopathology. 2011; 140:307-311.

2. Almeida NR, Danelli MGM, Silva LHP, Hagiwara MK; Mazur C.
Prevalence of feline leukemia virus infection in domestic cats in Rio de Janeiro. Journal of Feline Medicine and Surgery. 2012; 14(8):583-586

3. Levy J, Crawford PC, Hartmann K, Hofmann-Lehmann R, Little S, Sundahl E. American Association of Feline Practitioners' feline retrovirus management guidelines. Journal of Feline and Surgery. 2008; 10(3):300316

4. Hartmann K. Clinical aspects of feline immunodeficiency and feline leukemia virus infection. Veterinary Immunology and Immunopathology. 2011; 143:190- 201

5. Gomes-Keller MA, Tandon R, Gönczi E, Meli ML, Hofmann-Lehmann $\mathrm{R}$, Lutz H. Shedding of feline leukemia virus RNA in saliva is a consistent feature in viremic cats. Veterinary Microbiology. 2006; 112(1): 11-21.

6. Willett B, Holsie M.J. Feline leukaemia virus: Half a century since its discovery. The Veterinary Journal. 2013;195(1):16-23

7. Hoofmann-Fezer G, Mortelbauer W, Hartmann K, Mysliwietz J, Thefeld S, Beer B, Thum I, Kraft, W. Comparison of T-cell subpopulations in cats naturally infected with feline leukaemia virus or feline immunodeficiency virus. Research in Veterinary Science. 1996; 61(3):222-226.

8. Dunham SP, Graham E. Retroviral Infections of Small Animals. Veterinary Clinics of North America: Small Animals Practice. 2008;38:879-901.

9. Hoover EA, Mullins J.I, Quackenbush S.L. Experimental transmission and pathogenesis of immunodeficiency syndrome in cats. Blood. 1987;70(6):1880-1892.

10. Rojko JL, Hardy Jr WD. Feline Leukaemia virus and other retroviruses. In: SHERDING, R.G. The Cat: diseases and clinical management. New York. Churchill Livingstone, 1994, 2.ed, p. 263-432.

11. Stuetzer B, Brunner K, Lutz H, Hartmann K. A trial with 3'-azido2',3'- dideoxythymidine and human interferon- $\alpha$ in cats naturally infected with feline leukaemia virus. Journal of Feline Medicine and Surgery. 2013;15(8): 667-671.

12. Hagiwara MK, Reche Jr A, Lucas SRR. Estudo clínico da infecção de felinos pelo vírus da leucemia felina em São Paulo. Revista Brasileira de Ciência Veterinária. 1997; 4(1):35-38.

13. Macieira, DB, Menezes RCAA, Damico CB, Almosny NRP, Mclane HL, Daggy JK, Messick JB. Prevalence and risk factors for hemoplasmas in domestic cats naturally infected with feline immunodeficiency virus and/or feline leukemia virus in Rio de Janeiro - Brazil. Journal of Feline Medicine and Surgery. 2008;10:120-129.

14. NajafI H, Madadgar O, Jamshidi S, Langeroudi GA, Lemraski M.D. Molecular and clinical study on prevalence of feline herpesvirus type 1 and calicivirus in correlation with feline leukemia and immunodeficiency viruses. Veterinary Research Forum. 2014;5(4):255-261.

15. Weiss ATH, Klopfleisch R, Gruber DA. Prevalence of feline leukaemia provirus DNA in feline lymphomas. Journal of Feline Medicine and Surgery. $2010 \cdot 12 \cdot 929-935$

16. Crawford C. Progressos no diagnóstico de infeccões retrovirais. In: HARTMANN, K. Feline Leukemia Virus Infection. In: GREENE, C. E. Infectious Diseases of the Dog and Cat. 3ed., Philadelphia, WB Saunders Company, 2006. cap.13. p.105-130.

17. Meichner K, Kruse BD, Hirschberger J, Hartmann K. Changes in prevalence of progressive feline leukaemia virus infection in cats with lymphoma in Germany. Veterinary Record. 2012; 171(14): p348-348

18. Miyazawa, T. Infections of feline leukemia virus and feline immunodeficiency virus. Front Biosci. 2002;7:504-518

19. Torres AT, Mathiason CK, Hoover EA. Re-examination of feline leukemia virus: host relationships using real-time PCR. Virology. 2005; 332:272-283.

20. Tandon R, Cattori V, Willi B, Lutz H, Hofmann-Lehmann R. Quantification of endogenous and exogenous feline leukemia virus sequences by real-time PCR assays Veterinary Immunology and Immunopathology. 2008;123:129133

21. Hofmann-Lehmann R, Cattori V, Tandon R, Boretti FS, Meli ML, Riond $\mathrm{B}$, Lutz H. How molecular methods change our views of FeLV infection and vaccination. Veterinary Immunology and Immunopathology. 2008;123:119-123. 
22. JAIN, N.C. Essential of Veterinary hematology. Philadelphia: Lea and Febiger,1993. $417 \mathrm{p}$.

23. Nesina S, Helfer-Hungerbuehler A, Riond B, Boretti FS, Willi B, Meli ML, Grest P, Hofmann-Lehmann R. Retroviral DNA-the silent winner: blood transfusion containing latent feline leukemia provirus causes infection and disease in naïve recipient cats. Retrovirology. 2015;12:105.

24. Souza LL, Nobre MO, Silveira E, Reichak D, Nascente P. Esporotricose em gatos portadores do vírus da leucemia felina. Revista Brasileira de Ciência Veterinária, 2005;12(1-3):99-01.

25. Hardy WD Jr. Immunopathology induced by the feline leukemia virus. Springer Seminars in immunopathology. 1982;5(1):75-106.

26. Hoffmann-Jagielska M, Winncka A, Jagielski D, Micun J, Zmudzka M, Lechowski R. Influence of naturally acquired feline leukemia virus (FeLV) infection on the phagocytic and respiratory burst activity of neutrophils and monocytes of peripheral blood. Polish Journal of Veterinary Sciences. 2005;8(2):93-7.

27. Quackenbush SL, Donahue PR, Dean GA, Myles MH, Ackley CD, Cooper MD, Mullins JI, Hoover EA. Lymphocyte Subset Alterations and Viral Determinants of Immunodeficiency Disease Induction by the Feline Leukemia Virus FeLV-FAIDS. Journal of Virology. 1990;64(11):5465-74.

28. Lucas, SRR, Hagiwara MK, Reche Jr A, Germano PML. Ocorrência de anticorpos antitoxoplasma em gatos infectados naturalmente pelo vírus da imunodeficiência dos felinos. Brazilian Journal of Veterinary Research and Animal Science. 1998;35(1):41-5. 